\title{
Device for Hand Motor Rehabilitation, Using Grip Force Sensing
}

DOI: $10.46932 / \operatorname{sfjdv2n2-151~}$

Received in: March 1st, 2021

Accepted in: May 30th, 2021

\author{
Otto Fernando Proaño Arboleda \\ Bachelor of Science in Biomedical Engineering
}

Current Institution: Yachay Tech University - School of Biological Sciences \& Engineering Full address (Institutional): Hacienda San José s/n, Proyecto Yachay. 100115-Urcuquí. ECUADOR

E-mail: otto.proano@yachaytech.edu.ec

\section{Bryan Bolivar Cerón Andrade}

Bachelor of Science in Biomedical Engineering

Current Institution: Yachay Tech University - School of Biological Sciences \& Engineering Full address (Institutional): Hacienda San José s/n, Proyecto Yachay. 100115-Urcuquí. ECUADOR

E-mail: bryan.ceron@yachaytech.edu.ec

\section{Evelyn Cristina Picón Figueroa}

Bachelor of Science in Biomedical Engineering

Current Institution: Yachay Tech University - School of Biological Sciences \& Engineering Full address (Institutional): Hacienda San José s/n, Proyecto Yachay. 100115-Urcuquí. ECUADOR

E-mail: evelyn.picon@yachaytech.edu.ec

\begin{abstract}
Current treatments for rehabilitation of prehensile movements or recovery of grip strength are generally subjective, which means that the results are based on the perception of the physiotherapist who performs the treatment. However, there may be many reasons for altering these results, from errors in the perception to a possible misdiagnosis. Reason for which it is necessary to keep a record of the progress of recovery of grip strength. These records even make it possible to determine if the treatment is successful or quantify how much the patient is improving. Due to these problems, a biomedical device has been developed to measure and store the grip force in hand motor rehabilitation. It starts with an input device that measures the grip strength and stores this information in a database. These data are presented on a small screen for the patient and on a computer for the physiotherapist. According to the force exerted in the grip handle, the system measures the changes in grip force. It displays them on a different screen from the patient perspective and the physiotherapist's perspective. The developed device facilitates the follow-up and monitoring of results of hand motor rehabilitation for the specialist. It also involves the patient in active participation, improving the success rate in the recovery process.
\end{abstract}

Keywords: Rehabilitation, physical therapy, grip strength, hand disorders, biomedical devices.

\section{INTRODUCTION}

The human hand is the first tool used in physical contact with the environment. An anatomical and functional perspective shows an excellent capacity to develop multiple tasks with great precision (Leo et al., 2016). There are two types of hand movements, prehensile and non-prehensile actions. Prehensile 
activities are those that allow us to take and hold objects by gripping. Non-prehensile activities instead allow manipulating things by pushing or lifting (Fischer et al., 2014). The rehabilitation of prehensile movements (grip strength) will be the focus of our work.

A series of diseases or injuries, mainly muscles, and tendons can cause a decrease in the functions carried out by the hand. These complications can be due to congenital conditions, overuse injuries due to repetition of a particular movement, or trauma. Among the common diseases that affect grip strength are carpal tunnel syndrome (CTS) and hand-arm vibration syndrome (HAVS). CTS is caused by injury to the median nerve, where the nerve is trapped in the carpal tunnel passage (Kostopoulos \& Ghaly, 2018). Otherwise, HAVS is caused by prolonged agitation when using vibrating machinery and can be classified as neurological, vascular, and musculoskeletal disorders (Weir \& Lander, 2005). Both conditions present as a common symptom of loss of strength in the hands, making it difficult to grasp an object. Whatever the cause of the problem affecting grip strength, a rehabilitation process through physical therapy is required in advanced cases. Moreover, a study published by 'The Lancet' suggests that decreased grip strength is a simple and inexpensive indicator of a possible cardiovascular disease (Leong et al., 2015); reason for storing this grip force data could even be used to determine and stratify an individual's risk of cardiovascular death.

Habitually treatments used to restore grip mobility are subjective. According to these criteria, the treating physician determines the condition or progress of the patient. However, there is no a scale or a data record for follow-up. Nowadays, instruments that measure handgrip strength show a unique value and do not store this information in any way. Usually, this class of devices works mechanically since the measurement is based on dynamometers.

Driven by the causes mentioned, we have developed a device that makes it possible to quantitatively determine the value of the force exerted when gripping objects; useful in both diagnosis and treatment. This device can measure the progress in the rehabilitation of hand injuries, severe cases of CTS, HAVS, or similar pathologies where a weak grip exists. In cases like this, it is ideal for recording the patient's measurements to determine their progress or decline as part of the decision-making process. At the same time, our device seeks to encourage the patient to actively participate in their rehabilitation, thus improving the success rate in the recovery process (Arnetz et al., 2004).

\section{METHODS}

\subsection{INPUT DEVICES}

The device for hand motor rehabilitation is mainly based on using a force-sensing resistor (FSR) manufactured by Interlink Electronics Inc. The FSRs are resistors that change their value according to 
Figure 1. FSR 404 model.

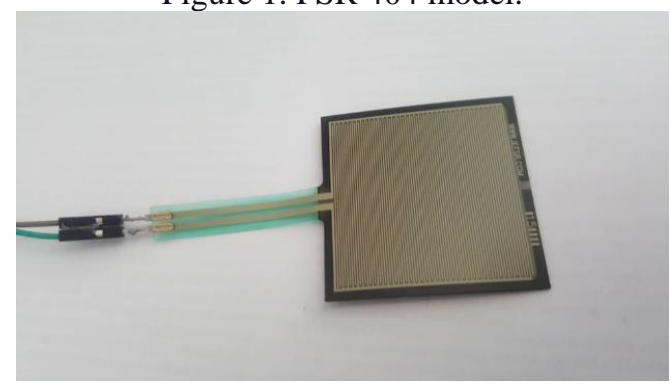

The FSR works in a voltage divider configuration which distributes the voltage (in this case, 5V) from a primary source among one or more connected impedances. Figure 2 shows the output voltage ( $V_{\text {out }}$ ) as a function of the applied force for different measuring resistor $\left(R_{M}\right)$ values. The value of the $R_{M}$ used was $10 \mathrm{k} \Omega$ because it generates a curve with better linearity compared to the others. This configuration allows an equation of the approximate graph to be found by interpolation. With this new equation, it is possible to approximate the force value (in grams) depending on the measured $\mathrm{V}_{\text {out }}$. Once the force measurements have been taken are transformed into kilograms and stored in a database.

Figure 2. Voltage divider of the FSR and the plot of output voltage $\left(\mathrm{V}_{\text {out }}\right)$ vs. applied force in grams for different measuring resistors $\left(\mathrm{R}_{\mathrm{M}}\right)$; extracted from the FSR 406 Data Sheet by Interlink Electronics Inc.
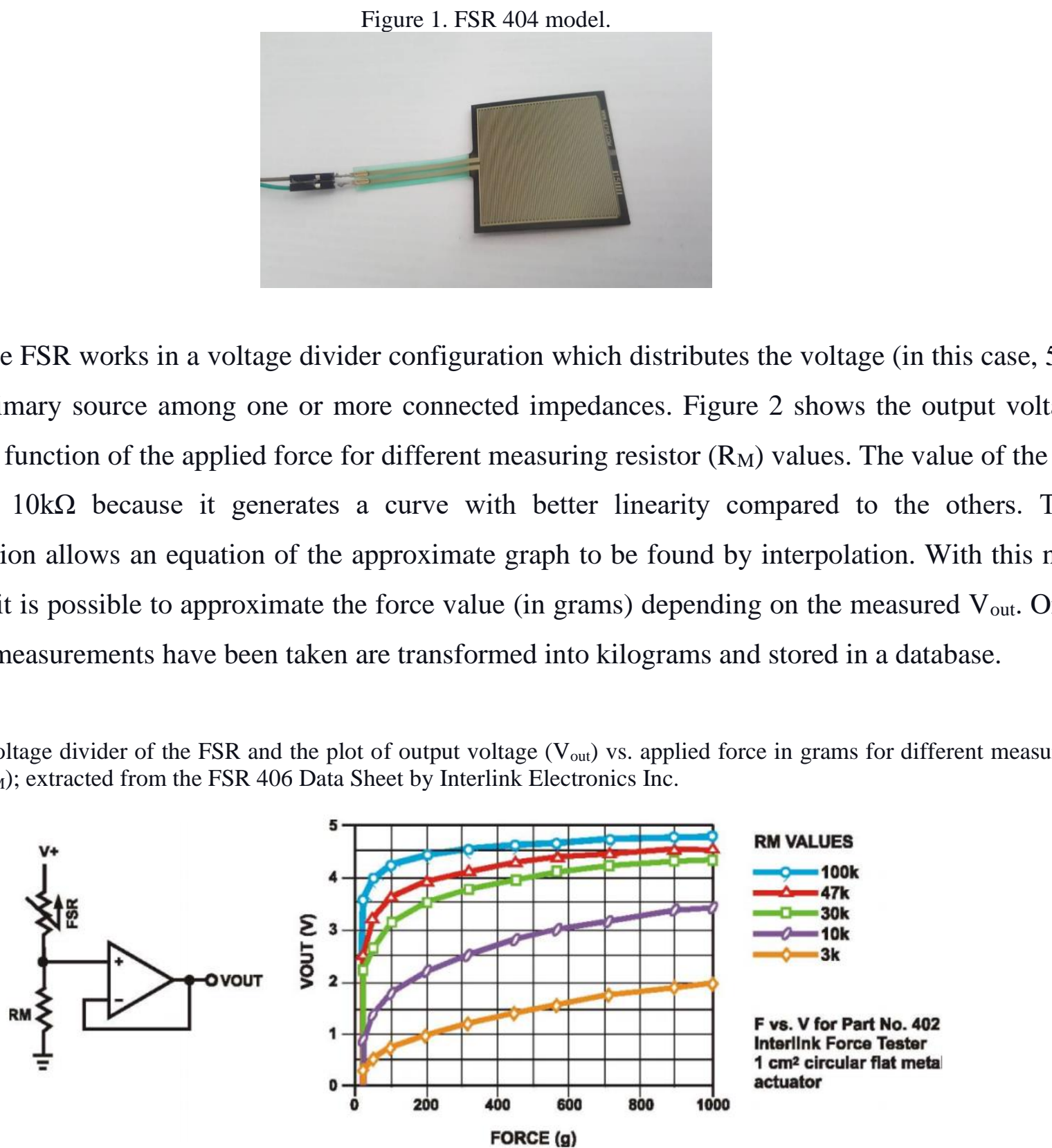

F vs. V for Part No. 402 Interlink Force Tester $1 \mathrm{~cm}^{2}$ circular flat meta actuator

Retrieved from: https://cdn.sparkfun.com/assets/c/4/6/8/b/2010-10-26-DataSheet-FSR406-Layout2.pdf

\subsection{OUTPUT DEVICES}

Otherwise, the output devices used where the results are shown were cataloged in two sections: the first one from the physiotherapist's perspective and the second from the patient's perspective. The patient will interact with the handle where the FSR is located, which will show its force value on an output 
screen (16x2 LCD) and a force indicator (LED sequence). The latter can be configured so that each LED can represent a specific force range to make a more user-friendly system for the patient (Figure 3).

Figure 3. Gripping handle, output screen, and the force indicator (Patient perspective).

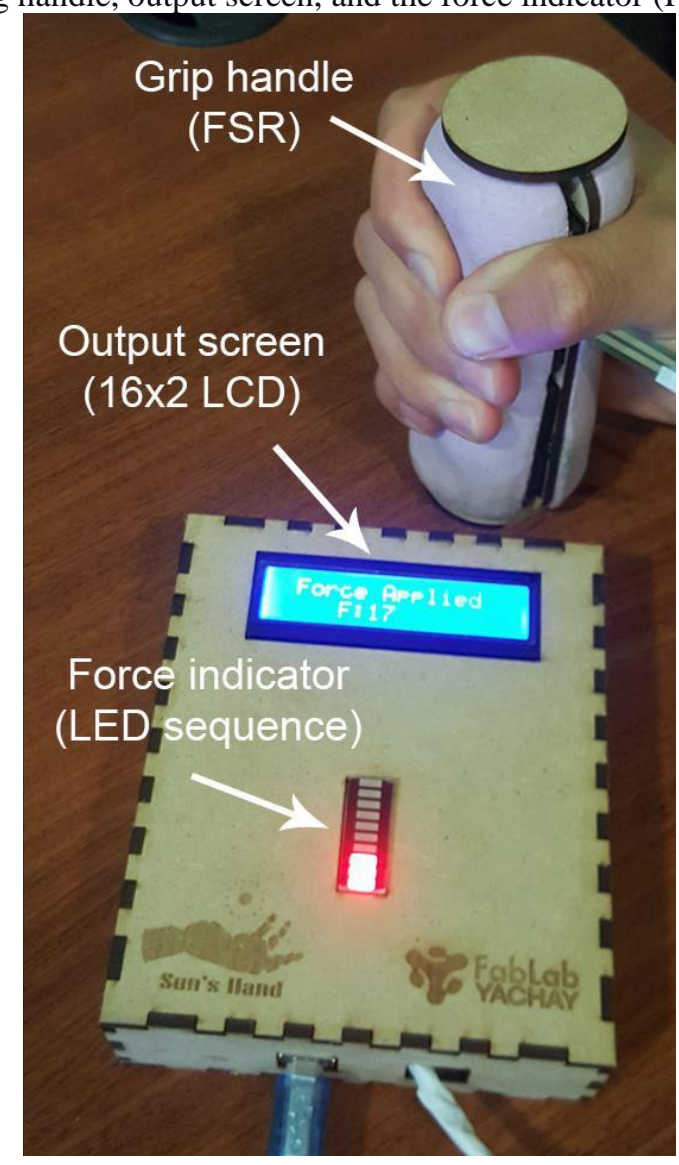

On the other hand, the results will be presented through a user interface on the physiotherapist's computer. All force data is stored in a database to be later reviewed by the specialist.

In this case, our main code and data processing center is located on an Arduino Uno. Further, to generate a connection from the Arduino one to a MySQL database, it was necessary to use the free software package XAMPP, which works as a PHP script interpreter and MySQL database management system. This connection establishes a connection bridge between our device and a MySQL database that stores the results. The stored data are shown in a user interface (Figure 4), which indicates the applied force at a specific time. This program was developed in Microsoft Visual Basic. 
Figure 4. The plot of force vs. time is shown in a computer (Physiotherapist perspective).

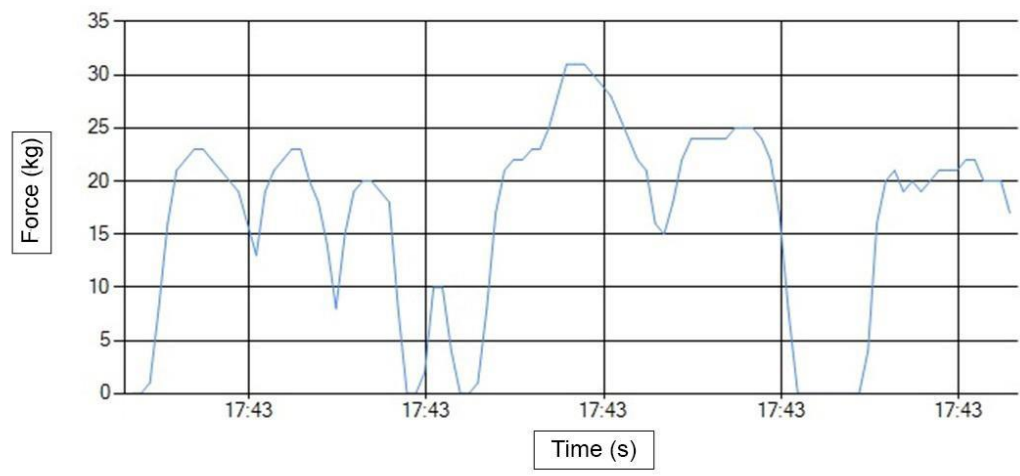

\subsection{GENERAL STRUCTURE OF THE DEVICE}

A general diagram of the operation of the grip force tracking device can be seen in Figure 5. It begins with FSR acting as the only input device to the circuit. The data of the measurements are processed in the main circuit board (Arduino Uno), where the input values detected are transformed into force values in kilograms. These force values are displayed in real-time on the output screen and the outside indicator for the patient. Although these output devices are very standard, the force indicator of the LED sequence has a particular function. Not only does it show the force exerted, but it attempts to generate a neurological response from the patient thanks to their active participation in the recovery process. At the same time, the force values are sent to a MySQL database where they are stored. This process is achieved through the XAMPP data management system. Here the data is displayed through a user interface that shows the results for the physiotherapist. 
Figure 5. General block diagram of the hand motor rehabilitation device operation.

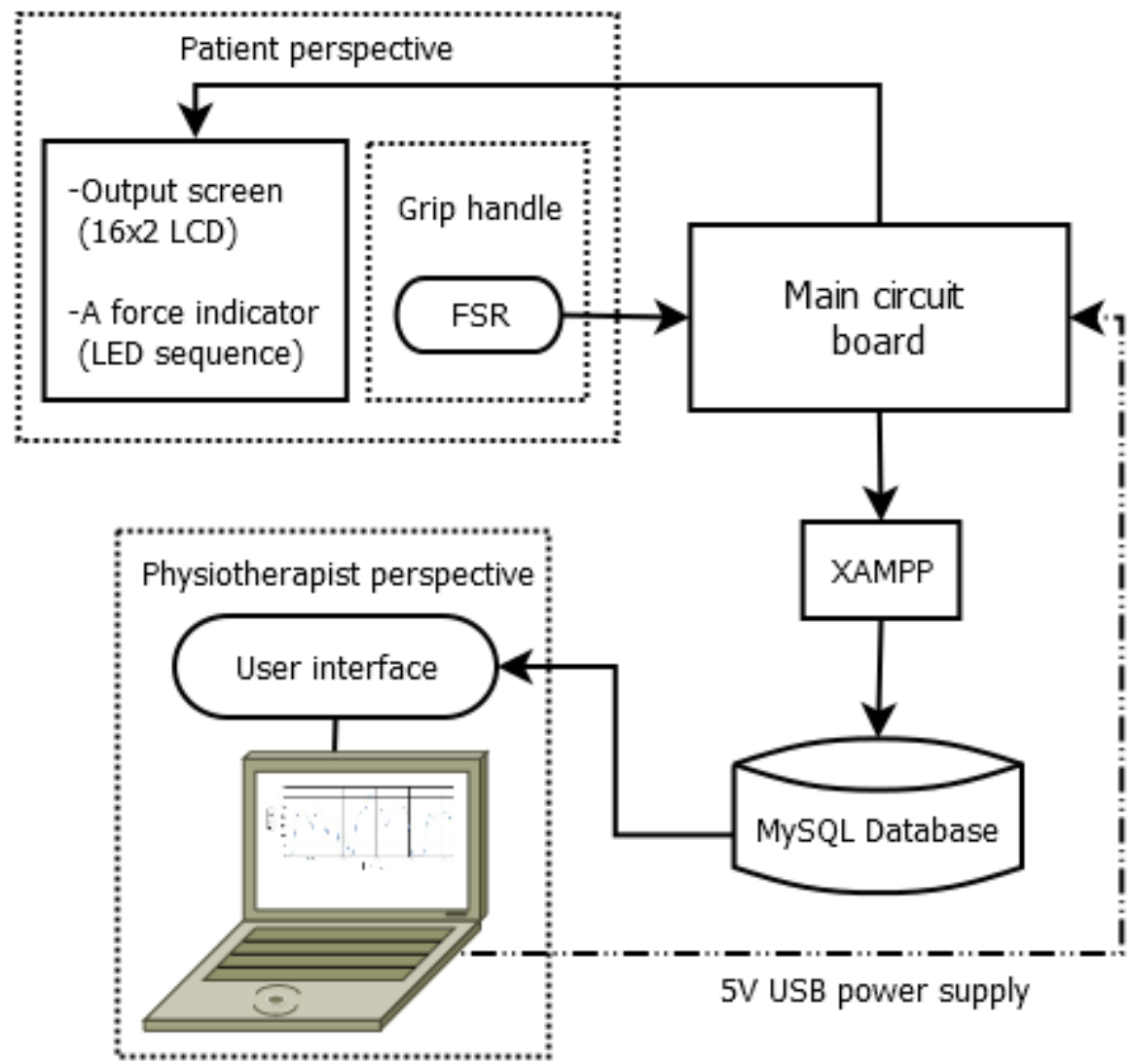

\section{RESULTS}

A prototype of the device for hand motor rehabilitation is already developed. The two sections of the device (patient perspective and physiotherapist perspective) work correctly. The system measures the changes in grip force according to the pressure exerted in the grip handle in a given time. From the patient's perspective, it is possible to actively participate in the recovery process by observing the results and progress. In addition, the grip handle was 3D printed to be more ergonomic and be more comfortable at the time of use.

Likewise, the applied force results that are stored make it easier for the physiotherapist to track the progress or decline of the physical therapy. It is also possible to measure the maximum grip force exerted by the patient and for how long at each appointment with the specialist.

\section{DISCUSSION}

In the present work, the design and implementation of a device to measure the gripping force during the concentric phase of the grip are proposed. Although still a prototype, our device is much smaller, simple to use, has a friendly style and encourages patient participation in rehabilitation, compared 
to its predecessors with rough designs, challenging to use and keep track of results. Despite though, it was a technological innovation at the time (Kurillo et al., 2005).

In the same way, unlike those grip force devices on the market, ours are electronic, not mechanical, allowing interaction in various ways with force measurements. For example, the output devices such as the screen indicate the force exerted in kilograms and the force indicator used to involve the patient in the recovery process. In other words, the patient can achieve a specific range of strength in their first rehabilitation appointments, and in the following sessions, they should try to overcome that range. These measurements can be helpful when evaluating the effectiveness of a treatment. Also, it is well known that the patient's active participation in a process results in a better response to rehabilitation treatment (Arnetz et al., 2004). Therefore, it seeks to meet this goal with the output screen and the force gauge for the patient's perspective.

This device is thought mainly to treat pathologies related to grip strength such as CTS, HAVS, lack of muscle tone, and loss of movement. Unfortunately, it has not yet been validated in actual patients, but the proposal of a device with these characteristics and the prototype shows enormous potential. In the future, the proper calibration of the FSR system (or even its replacement with better technology), calculation of errors, and validation of the device in actual clinical tests will be the new approaches. In addition, it seeks to improve the data management system by implementing a search by names or by an ID, graphs with timelines, and other display options with calculations of improvement percentages during treatment.

In conclusion, the device for hand motor rehabilitation works to track progress in the recovery of prehensile movements (grip strength) caused by illness or injury. At the same time, it helps the patient get involved and participate more in the recovery process. On the part of the physiotherapist, it gives a tool to monitor the treatment and support decision-making regarding the applied therapy. Although the prototype has not yet been validated with actual patients, it works correctly and shows great future potential.

\section{ACKNOWLEDGMENT}

We thank the Yachay Tech University and the School of Biological Sciences and Engineering for allowing us to use the laboratories to implement the project. We also appreciate the technical support of Prof. Graciela Salum, Ph.D. as a consultant and FabLab Yachay for the 3D printing of the grip handle and the case of the device. 


\section{FUNDING}

This research did not receive any specific grant from funding agencies in the public, commercial, or notfor-profit sectors.

\section{CONFLICT OF INTEREST}

Authors have no conflict of interest to declare. 


\section{REFERENCES}

Arnetz, J. E., Almin, I., Bergström, K., Franzén, Y., \& Nilsson, H. (2004). Active patient involvement in the establishment of physical therapy goals: Effects on treatment outcome and quality of care. Advances in Physiotherapy, 6(2), 50-69. https://doi.org/10.1080/14038190310017147

Fischer, J., Thompson, N. W., \& Harrison, J. W. K. (2014). The prehensile movements of the human hand. Classic Papers in Orthopaedics, 343-345. https://doi.org/10.1007/978-1-4471-5451-8_85

Kostopoulos, D., \& Ghaly, B. (2018). The use of electrodiagnostic studies and musculoskeletal sonography in carpal tunnel syndrome. Journal of Bodywork and Movement Therapies, 22(3), 718-719. https://doi.org/10.1016/j.jbmt.2018.07.001

Kurillo, G., Gregorič, M., Goljar, N., \& Bajd, T. (2005). Grip force tracking system for assessment and rehabilitation of hand function. Technology and Health Care, 13(3), 137-149. https://doi.org/10.3233/thc2005-13301

Leo, A., Handjaras, G., Marino, H., Bianchi, M., \& Moscatelli, A. (2016). Human and Robot Hands. Springer Series on Touch and Haptic Systems, 41-60. https://doi.org/10.1007/978-3-319-26706-7

Leong, D. P., Teo, K. K., Rangarajan, S., Lopez-Jaramillo, P., Avezum, A., Orlandini, A., Seron, P., Ahmed, S. H., Rosengren, A., Kelishadi, R., Rahman, O., Swaminathan, S., Iqbal, R., Gupta, R., Lear, S.

A., Oguz, A., Yusoff, K., Zatonska, K., Chifamba, J., ... Yusuf, S. (2015). Prognostic value of grip strength: Findings from the Prospective Urban Rural Epidemiology (PURE) study. The Lancet, 386(9990), 266-273. https://doi.org/10.1016/S0140-6736(14)62000-6

Weir, E., \& Lander, L. (2005). Hand-arm vibration syndrome. Cmaj, 172(8), 1001-1002. https://doi.org/10.1503/cmaj.045314 